\title{
Cytomegalovirus colitis and cow's milk allergy in an immunocompetent infant: is a causal or casual relationship?
}

\begin{abstract}
The association between cytomegalovirus (CMV) colitis and cow's milk allergy (CMPA) is rare and still unclear. The authors describe the case of an 8-week old female infant who presented with bloody diarrhea, lack of appetite, weight loss and fever due to CMV colitis. After some months she developed a CMPA. This case report describes the third case of CMV colitis associated with CMPA. This case strongly suggests a causal relationship between the two conditions.
\end{abstract}

Keywords: infant, cytomegalovirus, colitis, cow's milk allergy
Volume I Issue 3 - 2014

\author{
Luna Tanrikulu \\ Department of Dermatology, Zekai Tahir Burak Women?s \\ Health Training and Research Hospital,Turkey
}

Correspondence: Luna Tanrikulu, Department of Dermatology, Zekai Tahir Burak Women $\square$ s Health Training and Research Hospital,Ahmet Taner Kislali mah, Metissitesi I-Block No: 19, Cayyolu 068I0 Ankara, Turkey, Tel 905000000000 , Fax 903000000000, Email lunaderm@gmail.com

Received: May 24, 2014 | Published: July 05, 2014
Abbreviations: CMV, cytomegalovirus; CMPA, cow's milk allergy; PCR, polymerase chain reaction; ANA, anti-nuclear antibody; AMA, anti-microsomal antibody; ASMA, anti-smooth muscle antibody; ASCA, anti-saccharomyces cerevisiae antibody; ANCA, anti-neutrophil cytoplasmic antibody; ENA, anti-extractable nuclear antigens antibody

\section{Introduction}

Bloody diarrhoea in infants is an alarming symptom and requires further investigations. Cytomegalovirus (CMV) colitis is a cause of blood per rectum in combination with diarrhoea in infancy. ${ }^{1} \mathrm{CMV}$ colitis is one of the systemic manifestations of the CMV infection in immunocompromised hosts, while in immunocompetent hosts the $\mathrm{CMV}$ infection remains usually asymptomatic or, at most, manifests as non-specific viral syndrome or mononucleosis syndrome. Therefore, it has a benign and self-limiting course and rarely causes systemic manifestations. ${ }^{2}$ Infants are in the middle between immunocompetent and immunocompromised hosts because the immune system develops over the course of months of life and is influenced by breastfeeding mothers. Only few cases of CMV colitis in immunocompetent hosts have been previously reported in the literature. ${ }^{3-7}$ We describe a case of colitis due to primary postnatal CMV infection in an immunocompetent infant who manifested bloody diarrhea, lack of appetite and weight loss associated with fever. Interestingly, the CMV colitis was associated to the CMPA (cow's milk protein allergy). The possible relation between CMV colitis and CMPA is still unclear.

\section{Case presentation}

An 8-week old female infant, born at term with a weight of 2500 grams, was admitted to our Unit presenting with a 20 days history of bloody diarrhea, lack of appetite and weight loss associated with fever. She was born at term by vaginal delivery from non consanguineous parents, after a normal pregnancy. Child's mother anti-CMV IgM titre during pregnancy was negative. She was breast-fed for one month and then she was fed with a cow milk formula. Upon admission her weight was 2860 grams $\left(<3^{\circ}\right.$ percentile $)$ and the body temperature was $37.1^{\circ} \mathrm{C}$.
At the onset of the symptoms, she was hospitalized in another Pediatric Hospital, where she has been treated with nebicine, cefotaxime and, in suspect of a Kawasaki disease, intravenous immunoglobulines. This resulted in a temporary resolution of the symptoms, yet they appeared again after few days. At the admission at our unit skin turgor was slightly reduced, fontanelle was normal and the abdomen was slightly distended without hepatosplenomegaly and lymphadenopathy. We carried out a blood analysis that showed hemoglobin $9 \mathrm{gm} / \mathrm{dl}$, platelet count $1.227 .000 / \mathrm{mm}^{2}$, white blood cells $12.220 / \mathrm{mm}^{2}$ (neutrophils $46 \%$, lymphocytes $43 \%$, monocytes $9 \%$, eosinophils $1 \%$, basophils $1 \%$ ), C-reactive protein $3.71 \mathrm{mg} /$ $\mathrm{dl}$, erythrocyte sedimentation rate $120 \mathrm{~mm} / \mathrm{h}$, albumin $2.6 \mathrm{gm} / \mathrm{dl}$. Total serum IgE, transaminases, total proteins, urea, creatinine, immunoglobulin and complement were normal. Suspecting IgEmediated cow's milk allergy (CMPA), the determination of specific $\mathrm{IgE}$ and prick test were performed and they resulted normal. Lymphocyte subsets tests showed a slight reduction of CD8+ T cells. Cultures of blood, pharynx and urine were negative. Stool analysis was negative for Escherichia coli, Shighella spp., Yersinia enterocolitica, Clostridium difficile, Campylobacter spp., Amoebas, Parasites and Rotavirus, while the occult blood test was positive. Autoimmunity analysis resulted normal. Abdomen radiography showed a mild distension of intestinal loops, without signs of airfluid levels. Abdominal ultrasonography revealed the presence of a thin liquid film between the intestinal bends, and a thickening of the descending colon and sigma, without lymphadenomegaly and hepatosplenomegaly. Serologic examination and culture were negative. A limited colonoscopy showed a friable, ulcerated, hyperemic and edematous mucosa in the sigmoid, and the rectum with loss of the normal vascular pattern and ulcers covered with fibrin. The histological exam of multiple biopsies, obtained during colonoscopy, showed a diffuse atrophy of crypts with hyperplastic -regenerative features, a marked infiltrate of plasma cells and polymorphonuclear leucocytes in the lamina propria, cytonuclear eosinophylic inclusion bodies in endothelial and stromal cells.

All those features were compatible with the CMV infection, and this was later confirmed by polymerase chain reaction (PCR). Antibody titers analysis revealed that the anti-CMV IgG titer was 
44UA $/ \mathrm{ml}$ (with positive values $>6 \mathrm{UA} / \mathrm{ml}$ ), and the serum was positive for the presence of $\operatorname{IgM}(>0.9 \mathrm{TV})$. PCR for CMV on blood and urine resulted respectively $3.000 .000 \mathrm{gEq} / \mathrm{ml}$ and $190.000 \mathrm{gEq} / \mathrm{ml}$. Cerebral ecography, ocular examination and audiologic tests were normal. The mother was also tested for CMV and she had an IgG titer of $42 \mathrm{UA} / \mathrm{ml}$ and was negative for the presence of IgM. An intravenous treatment with ganciclovir was started, with an initial dose of $10 \mathrm{mg} / \mathrm{kg} / \mathrm{die}$ for 4 weeks. This resulted in a gradual resolution of the clinical symptoms. The patient diet was also redirected to a hydrolyzed formula (Neocate) because of the severity of intestinal mucosal damage. Neocate is a nutritionally complete, powdered amino-acid based exempt infant formula, indicated for CMPA and other gastrointestinal affections. She was discharged in good condition after one month.

After two months, the infant was hospitalized because she again developed irritability, colic, vomiting and diarrhea. Stool occult blood test and stool analysis for Bacteria, Parasites and Rotavirus were negative. Serology for CMV resulted $\mathrm{IgG} 30 \mathrm{UA} / \mathrm{ml}$ and $\operatorname{IgM}$ negative. Child's mother admitted that she had started cow's milk reintroduction (Humana 1) one week before. Humana 1 is infant milk used from birth to $60^{\circ}$ day of life it contains milk proteins, so it is contraindicated in child affected of CMPA. Therefore, the challenge test was considered positive. Considering the diagnosis of CMPA, we decided to reintroduce Neocate, and the symptoms resolved. One month after the reintroduction of Neocate the patient was in general good condition, her growth, clinical examination and neurodevelopment were normal.

\section{Discussion}

We describe the case of 8-week old infant with bloody diarrhea, lack of appetite, weight loss and fever caused by CMV. The diagnosis of CMV colitis was made by colonoscopy, after excluding all the other possible causes of bloody diarrhea in infants and persisting symptoms. Indeed, the performed analysis showed eosinophylic inclusion bodies in the endothelial and stromal cells, a typical sign of CMV infection. The detection of CMV DNA by PCR in blood, urine and intestinal biopsy tissue, associated with positive IgM, confirmed the diagnosis. Two months after the hospitalization a second diagnosis of CMPA was made due to the development of irritability, colic, vomiting and diarrhea after the reintroduction of cow's milk formula. According to ESPGHAN guidelines, the diagnosis of CMPA is based on clinical presentation, improvement following introduction of hypoallergenic formula, and worsening of clinical features after challenge with CMP. ${ }^{8}$ Accordingly, we can state that the case we present was an example of CMPA non-IgE mediated (prick test and specific test were negative) ${ }^{9}$

The association between CMV colitis and CMPA is rare, only two cases have been reported in immunocompetent infants, and the pathogenetic mechanism is still unknown. In the first case, described in 1997, a five week old male, breast-fed for 4 weeks, presented diarrhea, blood per rectum associated with vomit and weigh loss after 2 days from the introduction of a cow's milk formula. A diagnosis of CMPA was made, so he was fed with an extensively hydrolysed formula with improvement of symptoms and weight gain, even if intermittent minimal blood per rectum persisted. Because of persisting of blood per rectum, a limited colonoscopy was performed and showed a macroscopic colitis. Biopsies revealed inclusion bodies in epithelial and endothelial cells typical of CMV infection, and the diagnosis was confirmed by immunohistochemical staining. Throat and urine culture for CMV resulted positive, and antibodies titres for CMV revealed an $\mathrm{IgG}$ of $1.2 \mathrm{IU} / \mathrm{ml}$ and positive $\mathrm{IgM}$. The bleeding resolved with the resolution of CMV infection, while the patient was still fed with the extensively hydrolysed formula. At five months of life, a challenge with normal formula failed and CMPA was confirmed. ${ }^{10}$
The second case described a two-month old male who presented with a history of bloody mucous diarrhoea 6 days after the introduction of cow's milk formula. CMPA was suspected and breast feeding with exclusion of all cow's milk products from the mother's diet was suggested. As a consequence, the symptoms disappeared. At age of 3 months, one week after the reintroduction of cow's milk formula, he again developed blood per rectum and diarrhoea. In spite of being fed with an amino acid-based formula, his blood per rectum persisted. At that time, a diagnosis of perinatal CMV infection was made, based on CMV detection in the infant's blood and urine as well as in the maternal milk. Being exclusively fed with amino acid containing formula, one week later the blood per rectum stopped. ${ }^{11}$

The relation between CMV infection and CMPA is still unknown. Probably a disruption of gastrointestinal mucosa barrier by the CMPA could increase intestinal permeability leading to CMV infection. Indeed, immunocompetent infants develop CMV colitis when affected by underlying gastrointestinal disease: any mucosal injury can lead to an increase in the intestinal permeability, as well as an important $\mathrm{CMV}$ invasion. ${ }^{3}$ Furthermore, the continuous attack from CMV via breast-milk could aggravate the destruction of the normal gastrointestinal barrier by CMPA. The two cases reported in literature seem to confirm it. Another possibility is that CMV colitis causes colon damage and facilitates the development of CMPA. Even if the case we present seems to demonstrate this theory, we cannot certainly state that CMV colitis developed before CMPA. CMPA could have led to an asymptomatic inflammation and dysmotility of the gastrointestinal tract, which allowed CMV infection. The association between CMV gastrointestinal infection and CMPA remains unclear. This relation should be better investigated and discussed in order to understand if it only happens randomly or if there is a causal link. The case report we present seems to demonstrate a causal relationship between CMV colitis and CMPA. More studies are needed to elucidate the connection between these two conditions in infants with bloody diarrhoea.

\section{Acknowledgments}

None.

\section{Conflicts of interest}

The authors have no conflict of interests related to this publication and have not received any grants.

\section{Funding}

None.

\section{References}

1. Arvola $T$, Ruuska $T$, Keranen J, et al. Rectal bleeding in infancy: clinical, allergological, and microbiological examination. Pediatrics. 2006;117(4):e760-e768

2. Rafailidis PI, Mourtzoukou EG, Varbobitis IC, et al. Severe cytomegalovirus infection in apparently immunocompetent patients: a systematic review. Virol J. 2008;5:47.

3. Berardi A, Rossi C, Fiorini V, et al. Severe acquired cytomegalovirus infection in a full-term, formula-fed infant: case report. BMC Pediatr. 2011;11:52.

4. Cheong JL, Cowan FM, Modi N. Gastrointestinal manifestations of postnatal cytomegalovirus infection in infants admitted to a neonatal intensive care unit over a five year period. Arch Dis Child Fetal Neonatal Ed. 2004;89(4):F367-F369.

5. Buonuomo PS, Maurizi P, Valentini P, et al. Successful treatment with oral valganciclovir in immunocompetent infant with gastrointestinal manifestations of cytomegalovirus infection. $J$ Perinatol. 2006;26(10):648-649. 
6. Fox LM, Gerber MA, Penix L, et al. Intractable diarrhea from cytomegalovirus enterocolitis in an immunocompetent infant. Pediatrics. 1999;103(1):E10.

7. Hinds R, Brueton MJ, Francis N, et al. Another cause of bloody diarrhoea in infancy:cytomegalovirus colitis in an immunocompetent child. J Paediatr Child Health. 2004;40(9-10):581-582.

8. Koletzko S, Niggemann B, Arato A, et al. Diagnostic approach and management of cow's-milk protein allergy in infants and children: ESPGHAN GI Committee practical guidelines. J Pediatr Gastroenterol Nutr. 2012;55(2):221-229.
9. NIAID-Sponsored Expert Panel, Boyce JA, Assa'ad A, et al.Guidelines for the diagnosis and management of food allergy in the United States:report of the NIAID-sponsored expert panel. J Allergy Clin Immunol. 2010;126(6 Suppl):S1-S58.

10. Jonkhoff-Slok TW, Veenhoven RH, de Graeff-Meeder ER, et al. An immunocompetent infant with cow's milk allergy and cytomegalovirus colitis. Eur J Pediatr. 1997;156(7):528-529.

11. Rogalidou M, Roma E, Kouroumalis E, et al. Allergic colitis in an infant with perinatal cytomegalovirus infection. Ann Gastroenterol. 2010;23(2):139-141. 